\title{
$\eta$ CARINAE BABY HOMUNCULUS UNCOVERED BY ALMA
}

\author{
Zulema Abraham ${ }^{1}$, Diego Falceta-Gonçalves ${ }^{2,3}$, and Pedro P. B. Beaklini ${ }^{1,4}$ \\ ${ }^{1}$ Instituto de Astronomia, Geofísica e Ciências Atmosféricas, Universidade de São Paulo, R. do Matão 1226, Cidade Universitária, \\ CEP 05508-900 São Paulo, SP, Brazil; zulema.abraham@iag.usp.br \\ ${ }^{2}$ Escola de Artes, Ciências e Humanidades, Universidade de São Paulo, R. Arlindo Bettio 1000, 03828-000 São Paulo, SP, Brazil \\ Received 2014 April 6; accepted 2014 June 24; published 2014 July 31
}

\begin{abstract}
We report observations of $\eta$ Carinae obtained with ALMA in the continuum of 100, 230, 280, and $660 \mathrm{GHz}$ in 2012 November, with a resolution that varied from 2 .".8 to 0.45 for the lower and higher frequencies, respectively. The source is not resolved, even at the highest frequency; its spectrum is characteristic of thermal bremsstrahlung of a compact source, but different from the spectrum of optically thin wind. The recombination lines $\mathrm{H} 42 \alpha, \mathrm{He} 42 \alpha$, $\mathrm{H} 40 \alpha, \mathrm{He} 40 \alpha, \mathrm{H} 50 \beta, \mathrm{H} 28 \alpha, \mathrm{He} 28 \alpha, \mathrm{H} 21 \alpha$, and $\mathrm{He} 21 \alpha$ were also detected, and their intensities reveal non-local thermodynamic equilibrium effects. We found that the line profiles could only be fit by an expanding shell of dense and ionized gas, which produces a slow shock in the surroundings of $\eta$ Carinae. Combined with fittings to the continuum, we were able to constrain the shell size, radius, density, temperature, and velocity. The detection of the He recombination lines is compatible with the high-temperature gas and requires a high-energy ionizing photon flux, which must be provided by the companion star. The mass-loss rate and wind velocity, necessary to explain the formation of the shell, are compatible with an luminous blue variable eruption. The position, velocity, and physical parameters of the shell coincide with those of the Weigelt blobs. The dynamics found for the expanding shell correspond to matter ejected by $\eta$ Carinae in 1941 in an event similar to that which formed the Little Homunculus; for that reason, we called the new ejecta the "Baby Homunculus."
\end{abstract}

Key words: circumstellar matter - masers - stars: individual (Eta Carinae) - stars: mass-loss stars: winds, outflows

Online-only material: color figures

\section{INTRODUCTION}

The Homunculus Nebula, named by Gaviola (1950), is a bipolar shell of about $12 M_{\odot}$ of gas and dust (Morris et al. 1999; Smith et al. 2003), ejected from the massive star $\eta$ Carinae during the Great Eruption, in the decade of 1840. As the nebula expanded with an average velocity of $650 \mathrm{~km}$ $\mathrm{s}^{-1}$ to its actual size of about $18^{\prime \prime}$ or $0.089 \mathrm{pc}$ (assuming a distance to $\eta$ Carinae of $2.3 \mathrm{kpc}$ ), it cooled down forming a thick dust layer that absorbed the stellar light (Humphreys et al. 1999; Smith \& Gehrz 1998), slowly decreasing its luminosity and increasing the visual magnitude of $\eta$ Carinae from -1 in 1843 to 7.4 in 1887 . In that year, a new and sudden increase in the stellar luminosity was detected, which lasted for seven years and was interpreted as a new but minor episode of mass ejection, dimmed by dust absorption. The $0.1 M_{\odot}$ of ejected matter remained inside the Homunculus Nebula, and it is now at a distance of about \pm 2 " from the star, expanding at a velocity of about $200 \mathrm{~km} \mathrm{~s}^{-1}$; it was detected through long-slit mapping of several [Fe II] lines, using the Space Telescope Imaging Spectrograph, and named the Little Homunculus (LH) by Ishibashi et al. (2003).

The LH was also identified in the $8.6 \mathrm{GHz}(3 \mathrm{~cm})$ and $5.4 \mathrm{GHz}(6 \mathrm{~cm})$ radio continuum and hydrogen $(\mathrm{H} 91 \alpha$ and $\mathrm{H} 106 \alpha$ ) recombination line maps, obtained with the Australian Compact Array with a 1" resolution (Duncan et al. 1997; Smith 2005; Teodoro et al. 2008). On the other hand, observations at $230 \mathrm{GHz}(1 \mathrm{~mm})$ and $100 \mathrm{GHz}(3 \mathrm{~mm})$, obtained with the SEST radiotelescope with a resolution of $30^{\prime \prime}$ and $50^{\prime \prime}$, respectively,

\footnotetext{
3 Also at SUPA, School of Physics and Astronomy, University of St Andrews, North Haugh, St Andrews, Fife KY169SS, UK.

4 Also at IRAM, Institut de RadioAstronomie Millimétrique, 300 rue de la Piscine, Domaine Universitaire, F-38406 Saint Martin d'Hères, France.
}

showed a much stronger unresolved radio source at the star position, which was interpreted as free-free emission from an optically thick wind of spectral index 1.3 (Cox et al. 1995b). Recombination line observations $(\mathrm{H} 40 \alpha, \mathrm{H} 30 \alpha, \mathrm{H} 29 \alpha, \mathrm{H} 50 \beta$, and $\mathrm{H} 37 \beta$ ) at these wavelength bands showed strong emission at the compact source position, with evidence of departure from local thermodynamic equilibrium (NLTE), implying gas densities of more than $10^{7} \mathrm{~cm}^{-3}$ and electron temperatures of 15,000 K (Cox et al. 1995a).

The origin of the eruptions in $\eta$ Carinae is still under discussion; it includes luminous blue variable (LBV) behavior (Humphreys \& Davidson 1979), supernova impostors (Smith et al. 2011), or violent star collisions (Smith 2011). This last assumption is based on the binary nature of the $\eta$ Carinae system, revealed by the strict $5.54 \mathrm{yr}$ periodicity of the minima in the high-excitation line emission, X-ray, and radio continuum light curves (Damineli 1996; Corcoran et al. 2001; Abraham et al. 2005). The secondary star, in a highly eccentric orbit, has not been observed directly, but a range of possible surface temperatures, higher than that of $\eta$ Carinae, was determined through photoionization models (Mehner et al. 2010) of the high excitation lines in the Weigelt blobs (Weigelt \& Ebersberger 1986).

In this paper, we report continuum and $\mathrm{H}_{\mathrm{I}}$ and $\mathrm{He}_{\mathrm{I}}$ recombination line observations $(42 \alpha, 40 \alpha, 30 \alpha, 28 \alpha, 21 \alpha$, and $50 \beta)$ obtained with Atacama Large Millimeter/Submillimeter Array (ALMA) between 100 and $660 \mathrm{GHz}(3 \mathrm{~mm}$ and $455 \mu \mathrm{m}$, respectively), with resolutions ranging between 2 ".88 and 0'.45. These are the first observations of $\eta$ Carinae at such high frequencies and spatial resolutions. The observations show that the continuum spectrum has a turnover between 100 and $230 \mathrm{GHz}$, indicating that it arises in a compact $\mathrm{H}$ II region instead of an ionized wind. The recombination line intensities confirm the 
departure from LTE and the electron densities derived by Cox et al. (1995a) and constrain the geometry of the emitting region. In addition, the spectra show for the first time, besides for the $\mathrm{H}$ lines, the presence of the corresponding He I lines.

In Section 2, we describe the observations, and in Section 3, the continuum and recombination line maps, as well as the total spectrum of the region. In Section 4, we discuss the continuum spectrum and recombination line intensities and profiles and obtain the parameters of the emitting region, modeled as a spherical shell. Finally, in Section 5, we summarize our conclusions.

\section{OBSERVATIONS}

The observations were made with the ALMA as part of Early Science Cycle 0, on 2012 November 4 and 5, in four bands, centered at $92 \mathrm{GHz}(3.3 \mathrm{~mm}), 225 \mathrm{GHz}(1.3 \mathrm{~mm}), 291 \mathrm{GHz}$ $(1.0 \mathrm{~mm})$, and $672 \mathrm{GHz}(447 \mu \mathrm{m}) .^{5}$ The $7.5 \mathrm{GHz}$ bandwidth of the correlator was divided in four spectral windows, with a resolution of $488 \mathrm{kHz}$, which corresponds to $1.5,0.65,0.5$, and $0.22 \mathrm{~km} \mathrm{~s}^{-1}$ for each frequency band, respectively. This configuration allowed the observation of continuum emission as well as the recombination lines $\mathrm{H} 42 \alpha(85.7 \mathrm{GHz}), \mathrm{H} 40 \alpha(99.0 \mathrm{GHz})$, $\mathrm{H} 50 \beta(99.2 \mathrm{GHz}), \mathrm{H} 30 \alpha(231.9 \mathrm{GHz}), \mathrm{H} 28 \alpha(284.3 \mathrm{GHz})$, and $\mathrm{H} 21 \alpha(662.4 \mathrm{GHz})$. The corresponding $\mathrm{He} \alpha$ lines are separated from the $\mathrm{H} \alpha$ lines by $\Delta v / v=0.000407$, or $-122 \mathrm{~km} \mathrm{~s}^{-1}$, and were also present in the spectra. Twenty-three antennas were used in the observations, with a maximum baseline of $375 \mathrm{~m}$. The source J1107-448 was observed for bandpass calibration, J1038-5311 and Titan for phase calibration, and the latter also for flux calibration. The integration time for $\eta$ Carinae was $6 \mathrm{~s}$ for the lowest frequency bands and $18 \mathrm{~s}$ for the $672 \mathrm{GHz}$ band.

We used the CLEAN algorithm present in Common Astronomy Software Applications (CASA) to construct the images. The cell sizes used were $0.45,0.17,00^{\prime} 13$, and $00^{\prime} .05$ for the bands centered at 92, 225, 291, and $672 \mathrm{GHz}$, respectively. Continuum maps were built excluding the channels in which line emission was present. Once these maps were obtained, the channels with line emission were analyzed after subtracting the continuum contribution from the UV data. Line map cubes were obtained with a velocity resolution of $2 \mathrm{~km} \mathrm{~s}^{-1}$ for all lines, except for $\mathrm{H} 21 \alpha$ and $\mathrm{He} 21 \alpha$, for which the velocity resolution was $10 \mathrm{~km} \mathrm{~s}^{-1}$ to compensate for the low signal-to-noise ratio of the individual channels. All velocities in this paper refer to the local standard of rest (LSR).

Besides the ALMA continuum observations of $\eta$ Carinae, we used $43 \mathrm{GHz}$ data obtained with the Itapetinga ${ }^{6}$ radiotelescope, located in São Paulo, Brazil, on 2012 October 30. The characteristics of the receiver and the observing mode are described in Abraham et al. (2005).

\section{RESULTS}

In Figure 1, we show the continuum maps of $\eta$ Carinae for the four observed bands. The source remains unresolved at all frequencies. In Table 1, we present, for each band, the central frequency; the size and orientation of the beam; the flux

\footnotetext{
5 This paper makes use of the following ALMA data: ADS/JAO.ALMA\# 2011.0.00497.S_2012_12_20 and ADS/JAO.ALMA\#2011.0.00497.S_2013_01 29. ALMA is a partnership of ESO (representing its member states), NSF (USA), and NINS (Japan), together with NRC (Canada) and NSC and ASIAA (Taiwan), in cooperation with the Republic of Chile. The joint ALMA Observatory is operated by ESO, AUI/NRAO, and NAOJ.

6 The Itapetinga radiotelescope is operated by the Brazilian Space Agency Instituto de Pesquisas Espaciais (INPE).
}

Table 1

Continuum Parameters

\begin{tabular}{lcccc}
\hline \hline $\begin{array}{l}\text { Freq. } \\
(\mathrm{GHz})\end{array}$ & $\begin{array}{c}\text { Beam Size } \\
(\operatorname{arcsec})\end{array}$ & $\begin{array}{c}\text { P.A. } \\
(\mathrm{deg} .)\end{array}$ & $\begin{array}{c}\text { Max } \\
\left(\mathrm{Jy} \mathrm{beam}^{-1}\right)\end{array}$ & $\begin{array}{c}\text { Flux } \\
(\mathrm{Jy})\end{array}$ \\
\hline 43.0 & $144 \times 144$ & & & $8.7 \pm 1.0$ \\
92.5 & $2.88 \times 1.83$ & 54 & 20.9 & $28.4 \pm 0.03$ \\
225.4 & $1.52 \times 0.75$ & 62 & 26.2 & $41.2 \pm 0.06$ \\
291.2 & $0.83 \times 0.60$ & 35 & 25.7 & $44.3 \pm 0.10$ \\
671.7 & $0.45 \times 0.32$ & 42 & 22.1 & $40.2 \pm 0.09$ \\
\hline
\end{tabular}

Table 2

H Line Parameters

\begin{tabular}{lcccc}
\hline \hline Line & $\begin{array}{c}\text { Freq. } \\
(\mathrm{GHz})\end{array}$ & $\begin{array}{c}\text { Resolution } \\
\left(\mathrm{km} \mathrm{s}^{-1}\right)\end{array}$ & $\begin{array}{c}\text { Max. Flux } \\
(\mathrm{Jy})\end{array}$ & $\begin{array}{c}\text { Width } \\
\left(\mathrm{km} \mathrm{s}^{-1}\right)\end{array}$ \\
\hline $\mathrm{H} 42 \alpha$ & 85.69 & 2 & 12 & 29 \\
$\mathrm{H} 40 \alpha$ & 99.02 & 2 & 14 & 30 \\
$\mathrm{H} 30 \alpha$ & 231.92 & 2 & 75 & 36 \\
$\mathrm{H} 28 \alpha$ & 285.25 & 2 & 89 & 42 \\
$\mathrm{H} 21 \alpha$ & 662.40 & 10 & 150 & 40 \\
\hline
\end{tabular}

density of the peak, in Jy beam ${ }^{-1}$; the total flux density; and its uncertainty.

Maps in the radio recombination lines are similar to the continuum maps and are not shown in this paper. The line profiles, integrated over the whole emission region, are shown in Figure 2. They include the lines $\mathrm{H} 42 \alpha, \mathrm{He} 42 \alpha, \mathrm{H} 40 \alpha, \mathrm{He} 40 \alpha$, $\mathrm{H} 50 \beta, \mathrm{H} 28 \alpha, \mathrm{He} 28 \alpha, \mathrm{H} 21 \alpha$, and $\mathrm{He} 21 \alpha$. The frequencies were converted into velocities of the corresponding $\mathrm{H} \alpha$ lines; in such configurations, the He $\alpha$ lines appear separated by about $-122 \mathrm{~km} \mathrm{~s}^{-1}$ from the peak of the $\mathrm{H} \alpha$ lines and the $\mathrm{H} 50 \beta$ line by $-613 \mathrm{~km} \mathrm{~s}^{-1}$ from the $\mathrm{H} 40 \alpha$ line. The profiles were superimposed in Figure 3 in the velocity range $-300 \mathrm{~km} \mathrm{~s}^{-1}$ to $100 \mathrm{~km} \mathrm{~s}^{-1}$ to show the difference in flux density and width at half maximum between them. In Table 2, we present the frequency of the lines, the flux density, velocity of the peak, and the line width. The central velocity (referring to the LSR) varies between $-54 \mathrm{~km} \mathrm{~s}^{-1}$ and $-56 \mathrm{~km} \mathrm{~s}^{-1}$, while the systemic velocity of the $\eta$ Carinae, derived from the velocity of the Homunculus lobes is $-19.7 \mathrm{~km} \mathrm{~s}^{-1}$ (Smith 2004). The spectra clearly extend, with low intensity, from $+100 \mathrm{~km}^{-1}$ to $-500 \mathrm{~km}$ $\mathrm{s}^{-1}$. The line width varies between $29 \mathrm{~km} \mathrm{~s}^{-1}$ for the $\mathrm{H} 42 \alpha$ line to $40 \mathrm{~km} \mathrm{~s}^{-1}$ for the $\mathrm{H} 21 \alpha$ line, larger than the expected thermal velocity.

\section{DISCUSION}

\subsection{The Continuum Spectrum}

As shown in Table 1, the continuum flux density of $\eta$ Carinae increases with frequency up to $291 \mathrm{GHz}$ and slowly decreases afterward. This spectrum in typical of bremsstrahlung from compact $\mathrm{H}$ II regions. Since the source is not resolved in our observations, even at $670 \mathrm{GHz}$, we assumed spherical symmetry and solved the radiative transfer equation for a shell of radius $R$, width $\Delta R$, and constant density and temperature. We added to the four frequencies observed by ALMA, a $43 \mathrm{GHz}(7 \mathrm{~mm})$ observation obtained at the same epoch with the Itapetinga radiotelescope (2!.4 resolution), which gave a flux density of $8.7 \pm 1 \mathrm{Jy}$.

To calculate the flux density at each frequency band, we divided the source in rectangular cells of size $(\Delta x, \Delta y)$ in the plane of the sky and solved the radiation transfer equation along the line of sight ( $z$ coordinate) in each cell, using the continuum 

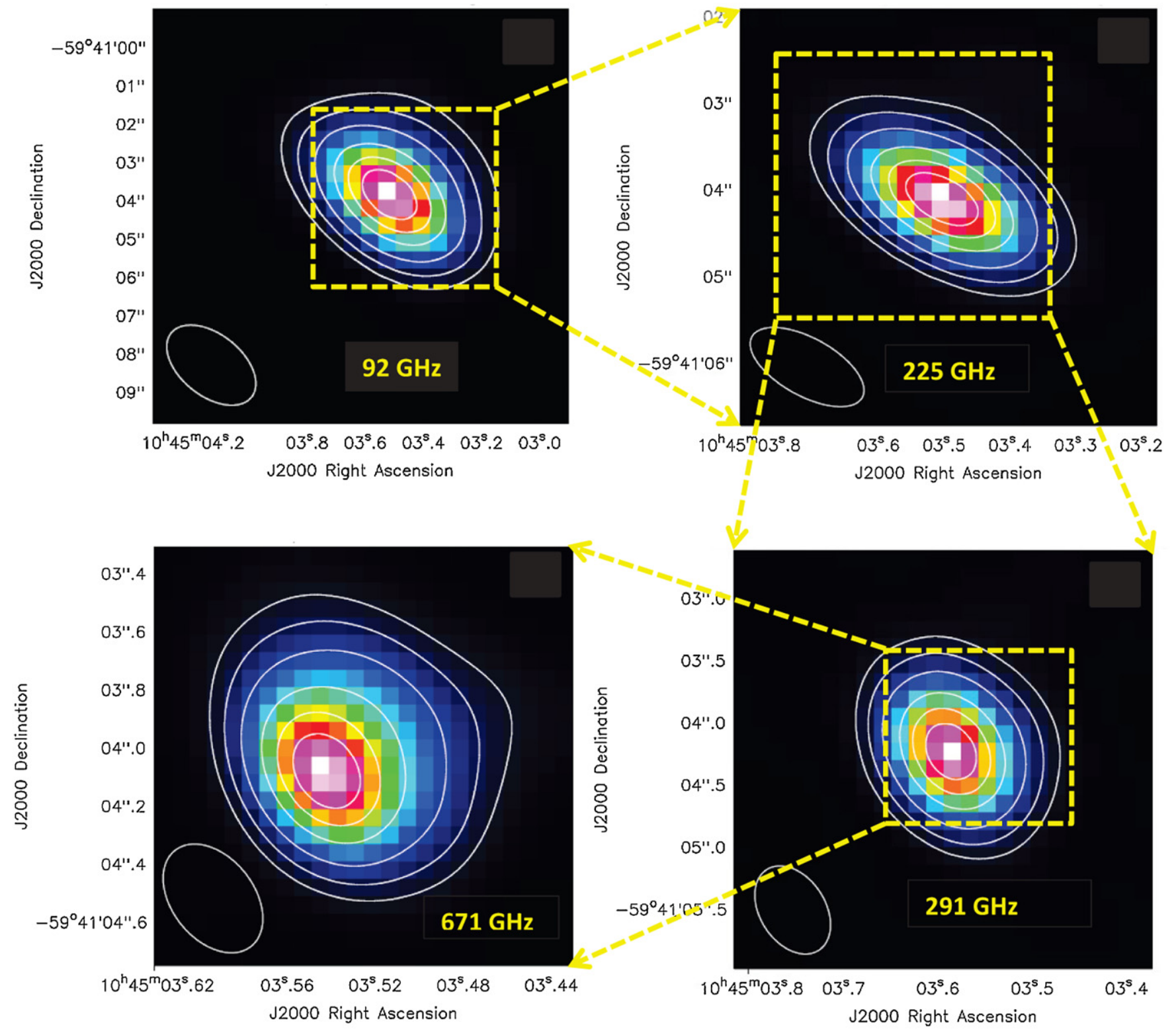

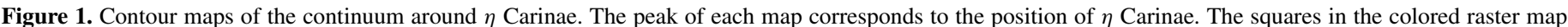

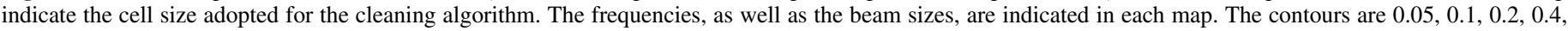

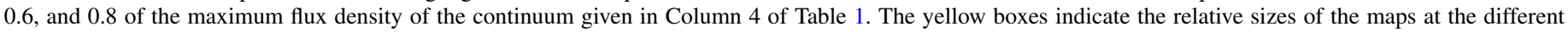
frequencies.

(A color version of this figure is available in the online journal.)

free-free emissivity equation (see Appendix A). We assumed that $\mathrm{H}$ is completely ionized and $\mathrm{He}$ is in the form of $\mathrm{He}^{+}$, implying that $N_{i}=N_{e}$. We integrated the flux density of each cell $S_{v}^{c}(x, y)$ over the emitting region using as parameters the relative shell width $\Delta R / R$ and the electron temperature $T_{e}$, and we determined the electron density and radius necessary to fit the observed spectrum. Good fits were obtained for all combinations of electron temperature and shell width; in Figure 4, we show the electron density and radius versus the relative shell widths $\Delta R / R$ for three values of $T_{e}: 10^{4}, 1.5 \times 10^{4}$, and $2 \times 10^{4} \mathrm{~K}$. We can see that the radius of the emitting region varied between a maximum value of $2.9 \times 10^{-3} \mathrm{pc}$ for $T_{e}=10^{4} \mathrm{~K}$ and $\Delta R / R=0.05$ and a minimum of $1.9 \times 10^{-3} \mathrm{pc}$ for $T=2 \times 10^{4} \mathrm{~K}$ and $\Delta R / R=1$. These dimensions correspond, at the distance of $\eta$ Carinae, to 0 .'26 and 0 '. 17, respectively, both smaller than the resolution of our observation. Two examples of continuum spectrum fittings are presented in Figure 5; they correspond to $T_{e}=1.5 \times 10^{4} \mathrm{~K}$ and $\Delta R / R=0.1$ and 1 , respectively.

\subsection{Recombination Line Emission}

To solve the transfer equation for line emission, it is necessary to take into account other parameters, namely, the thermal, turbulent, and bulk velocities, as well as the line profiles (a detailed description of the calculations is given in Appendix B).

To determine the velocity distribution of the different cells from the observations, we constructed the position-velocity diagram for all the $\mathrm{H}$ lines in the direction of the clean beam major axis, integrated by five pixels in the direction of the minor axis. In Figure 6, we show as an example the diagram for the $\mathrm{H} 28 \alpha$ line. We can see that the velocity distribution is compatible with that of a plasma cloud that has a velocity dispersion of 

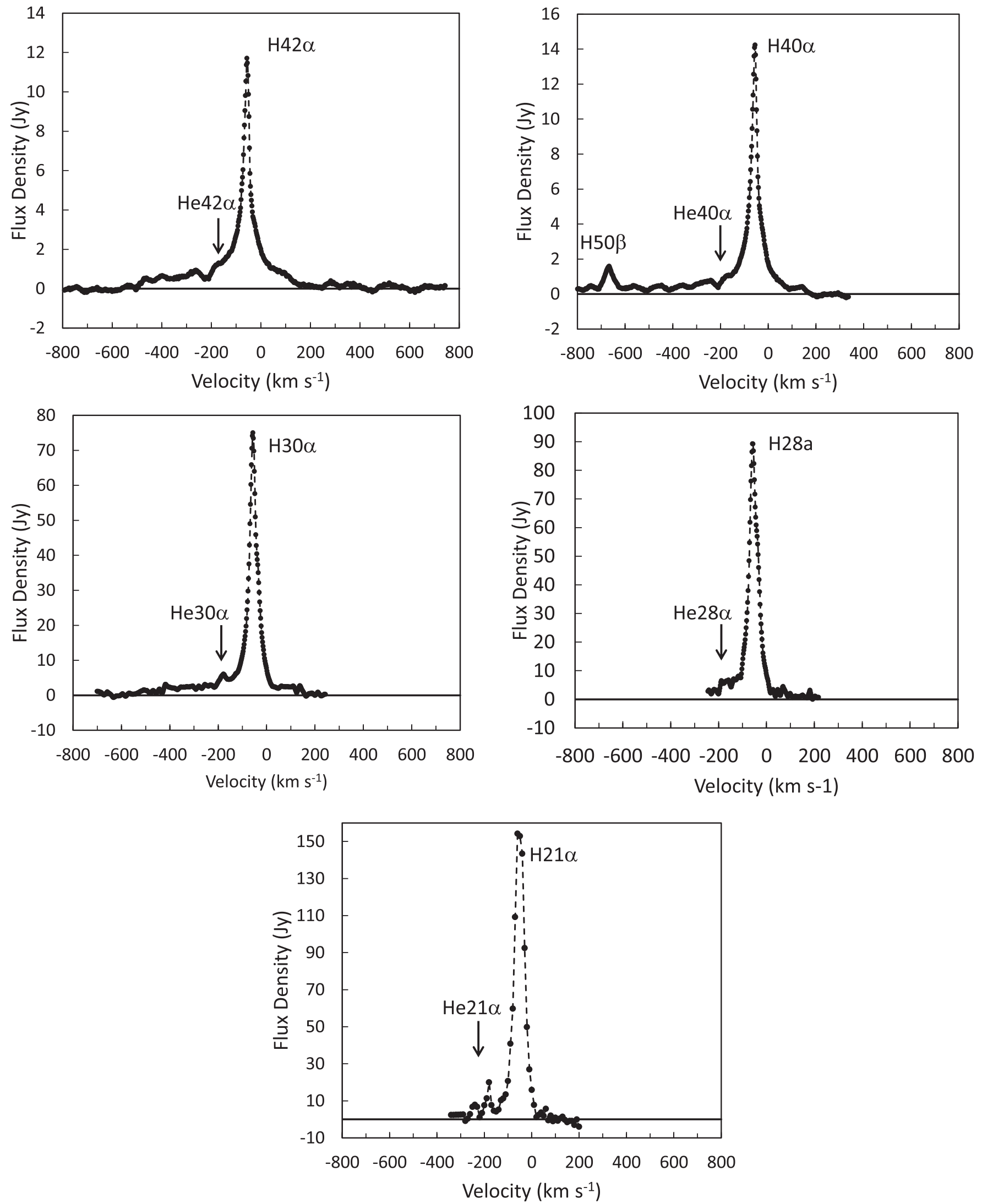

Figure 2. $\mathrm{H}$ and $\mathrm{He}$ recombination line spectra integrated over the emission maps. The spectral resolution is $2 \mathrm{~km} \mathrm{~s}^{-1}$ for all lines except the $\mathrm{H} 21 \alpha$ and $\mathrm{He} 21 \alpha$, for which the spectral resolution is $10 \mathrm{~km} \mathrm{~s}^{-1}$. 


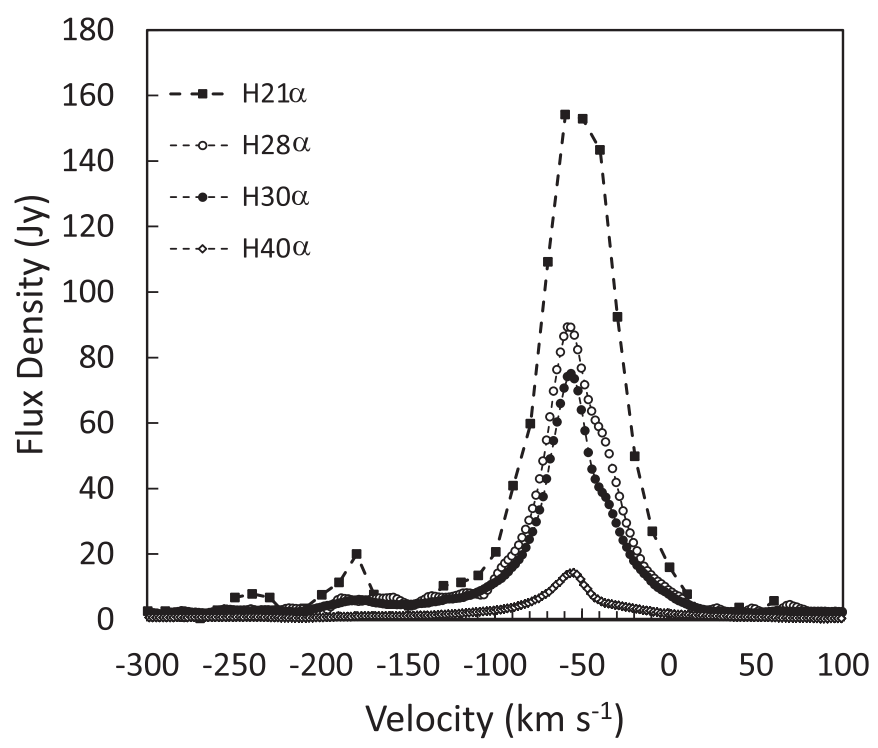

Figure 3. Superimposed $\mathrm{H}$ and $\mathrm{He}$ recombination line spectra $(40 \alpha, 30 \alpha, 28 \alpha$, and $21 \alpha$ ) integrated over the entire emission map.
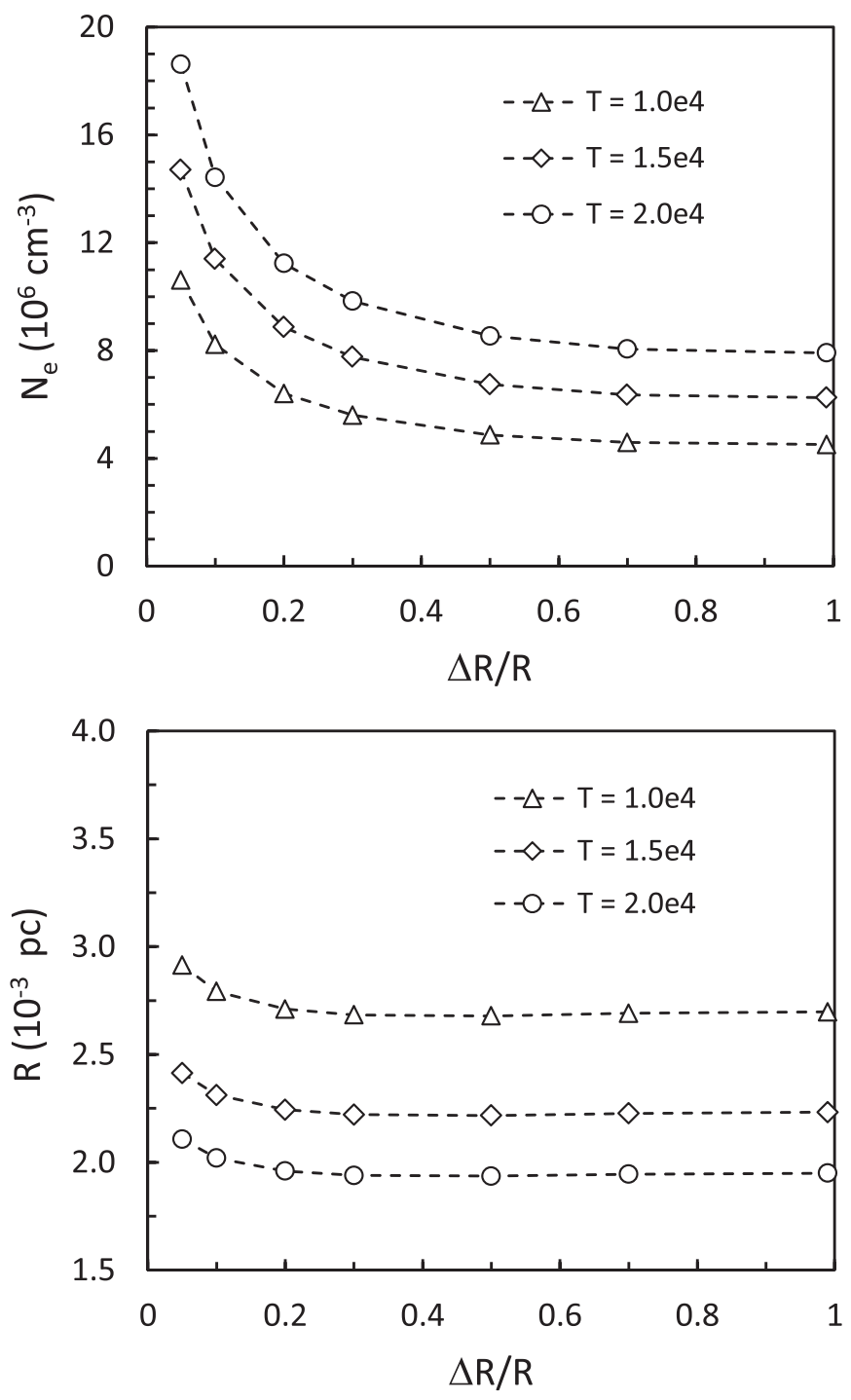

Figure 4. Electron density $N_{e}$ (top) and radius of the emitting region $R$ (bottom) as a function of the relative shell size $\Delta R / R$ for all the models that fit the continuum spectrum for three values of the electron temperature $T_{e}: 10^{4} \mathrm{~K}$, $1.5 \times 10^{4} \mathrm{~K}$, and $2 \times 10^{4} \mathrm{~K}$.
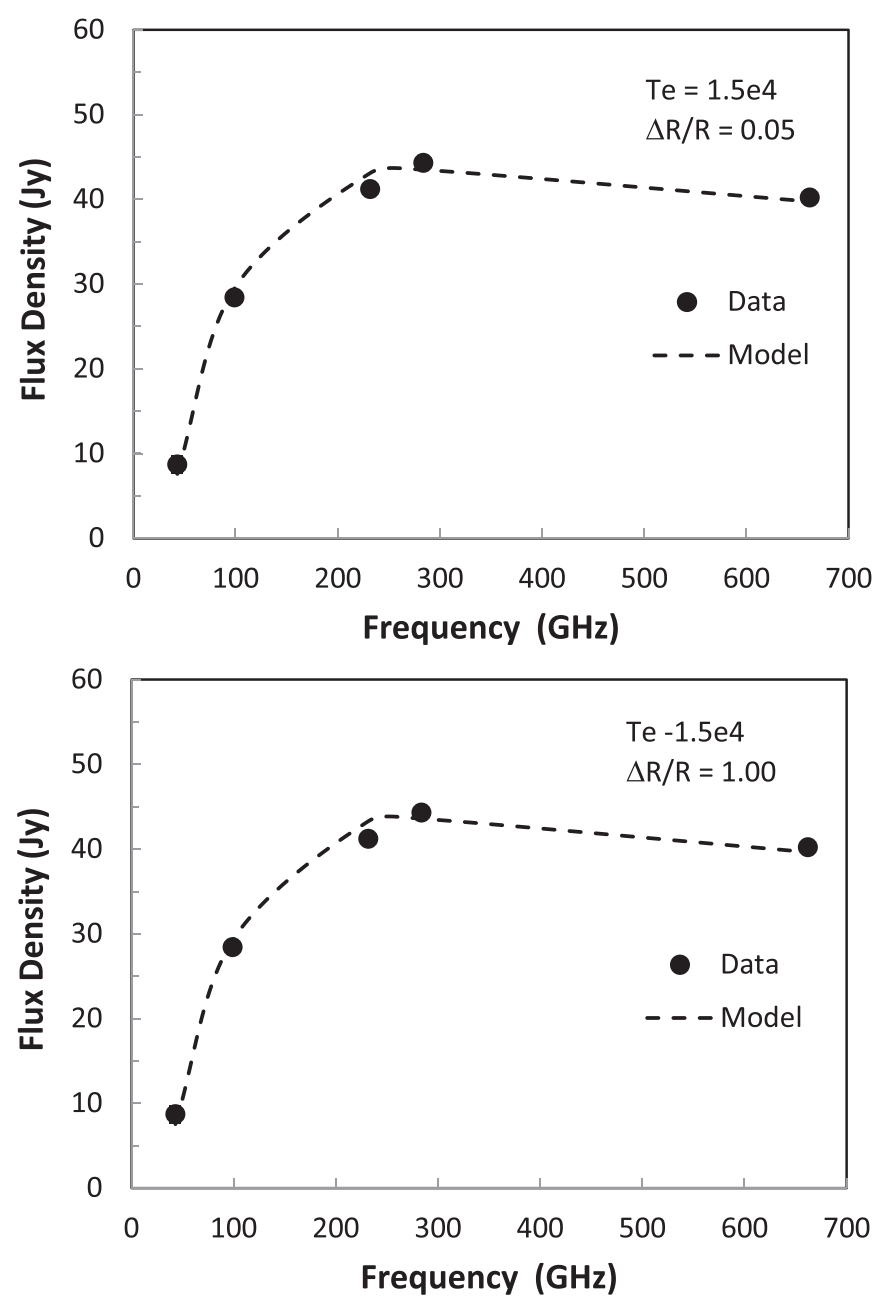

Figure 5. Observed continuum flux density (points) and models (broken lines) for an electron temperature of $1.5 \times 10^{4} \mathrm{~K}$, the corresponding electron density and radius given in Figure 4, and two values of the shell width $\Delta R / R: 0.05$ (top) and 1.0 (bottom).

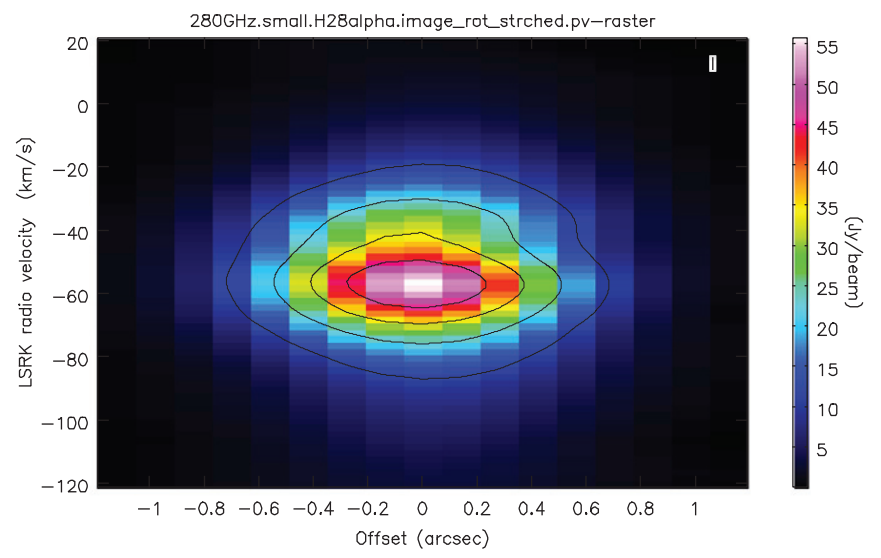

Figure 6. Position velocity diagram for the $\mathrm{H} 28 \alpha$ line in the direction of the clean beam major axis, integrated by five pixels in the direction of the minor axis. The contours are $0.2,0.4,0.6$, and 0.8 of the maximum flux density, which is $55.9 \mathrm{Jy}_{\text {beam }}{ }^{-1}$.

(A color version of this figure is available in the online journal.)

about $40 \mathrm{~km} \mathrm{~s}^{-1}$ and is moving with a bulk velocity of about $-56 \mathrm{~km} \mathrm{~s}^{-1}$.

With this information, we solved the transfer Equation (B1) and integrated the resulting flux density over the emitting region, 
using the combination of $N_{e}$ and $R$ obtained from the fitting of the continuum spectrum, for several combinations of $T_{e}$ and $\Delta R / R$. We were not able to reproduce the line profiles adding only turbulence to the thermal velocity, for that reason we assumed $T_{D}=T_{e}$ in Equation (B6) and introduced an expansion velocity with a linear gradient across the shell. We obtained the best fitting for an expansion velocity of $60 \mathrm{~km} \mathrm{~s}^{-1}$ in the internal part of the shell and of $20 \mathrm{~km} \mathrm{~s}^{-1}$ in the external part. To obtain the physical parameters of the emitting region, we calculated the ratio of the model and observed peak flux densities for the recombination lines $\mathrm{H} 40 \alpha, \mathrm{H} 30 \alpha, \mathrm{H} 28 \alpha$, and $\mathrm{H} 21 \alpha$ for values of $T_{e}$ ranging from $1.4 \times 10^{4} \mathrm{~K}$ to $2 \times 10^{4} \mathrm{~K}$ and for three values of $\Delta R / R: 0.05,0.1$, and 0.2 . The results are presented in Figure 7 , where we can see that the ratio becomes close to unity for all lines for $T_{e} \sim 1.7 \times 10^{4}$ and $\Delta R / R \sim 0.1$.

In Figure 8, we present the observed and model line profiles for $T=1.68 \times 10^{4} \mathrm{~K}$ and a relative shell width $\Delta R / R=0.1$. The other parameters of the fitting are $N_{e}=1.25 \times 10^{7} \mathrm{~cm}^{-3}$ and $R=2.2 \times 10^{-3} \mathrm{pc}$, values that also fit the continuum spectrum. We also show the observed and model line profiles for $\mathrm{H} 50 \beta$ and for all the He recombination lines, as well as all the $\mathrm{H}$ line profiles calculated assuming LTE. In all cases, the bulk velocity of the model is $-52 \mathrm{~km} \mathrm{~s}^{-1}$.

We can see that the model reproduces very well the highfrequency recombination line profiles $(\mathrm{H} 21 \alpha, \mathrm{H} 28 \alpha$ and $\mathrm{H} 30 \alpha)$. The fitting is not so good for the $\mathrm{H} 40 \alpha$ and $\mathrm{H} 42 \alpha$ lines, but we have to take into account that the continuum is optically thick at these frequencies, and in NLTE conditions, the line amplification, being proportional to $\tau_{v}^{l}+\tau_{v}^{c}<0$, is very sensitive to the value of $\tau_{v}^{c}>1$. Therefore, variations of the electron density across the shell or departure from spherical symmetry in the emitting region will more greatly affect the low-frequency lines than the high-frequency lines, for which $\tau_{v}^{c} \ll 1$. Besides, low-intensity line emission is seen in the $\mathrm{H} 40 \alpha$ and $\mathrm{H} 42 \alpha$ lines extending up to velocities of $-500 \mathrm{~km} \mathrm{~s}^{-1}$; they are probably produced in the LH and can absorb part of the low-velocity line emission. In the case of departure from spherical symmetry, the velocity distribution necessary to fit the line profiles will depend on the density and temperature distributions, and the solution will be degenerate. A more realistic model could be developed once data with finer angular resolution become available.

\subsection{The "Baby Homunculus"}

In the previous section, we showed that the continuum spectrum and recombination line profiles of $\eta$ Carinae can originate in a spherical ionized shell of radius $R=6.6 \times$ $10^{15} \mathrm{~cm}$ and width $0.1 R$, equivalent to $0^{\prime \prime} .2$ and $0^{\prime \prime} .02$ in the plane of the sky, respectively. The shell has constant density $N_{e}=1.27 \times 10^{7} \mathrm{~cm}^{-3}$ and temperature $T_{e}=1.7 \times 10^{4} \mathrm{~K}$, and it is moving with a bulk velocity of $-52 \mathrm{~km} \mathrm{~s}^{-1}$ in the LSR velocity frame and expanding with a velocity that varies linearly across the shell between $60 \mathrm{~km} \mathrm{~s}^{-1}$ in the inner border of the shell and $20 \mathrm{~km} \mathrm{~s}^{-1}$ in the outer border. This velocity gradient, together with the high gas temperature, suggests the existence of a slow shock, produced by the interaction of the expanding shell and the surrounding media. However, the ionizing source must be the secondary star since the radio light curves present the same $5.5 \mathrm{yr}$ periodic behavior observed at optical wavelengths and at X-rays (Cox et al. 1995b; Abraham et al. 2005). The observed He recombination lines indicate that the ionizing star must have a surface temperature higher than that of $\eta$ Carinae (Mehner et al. 2010).
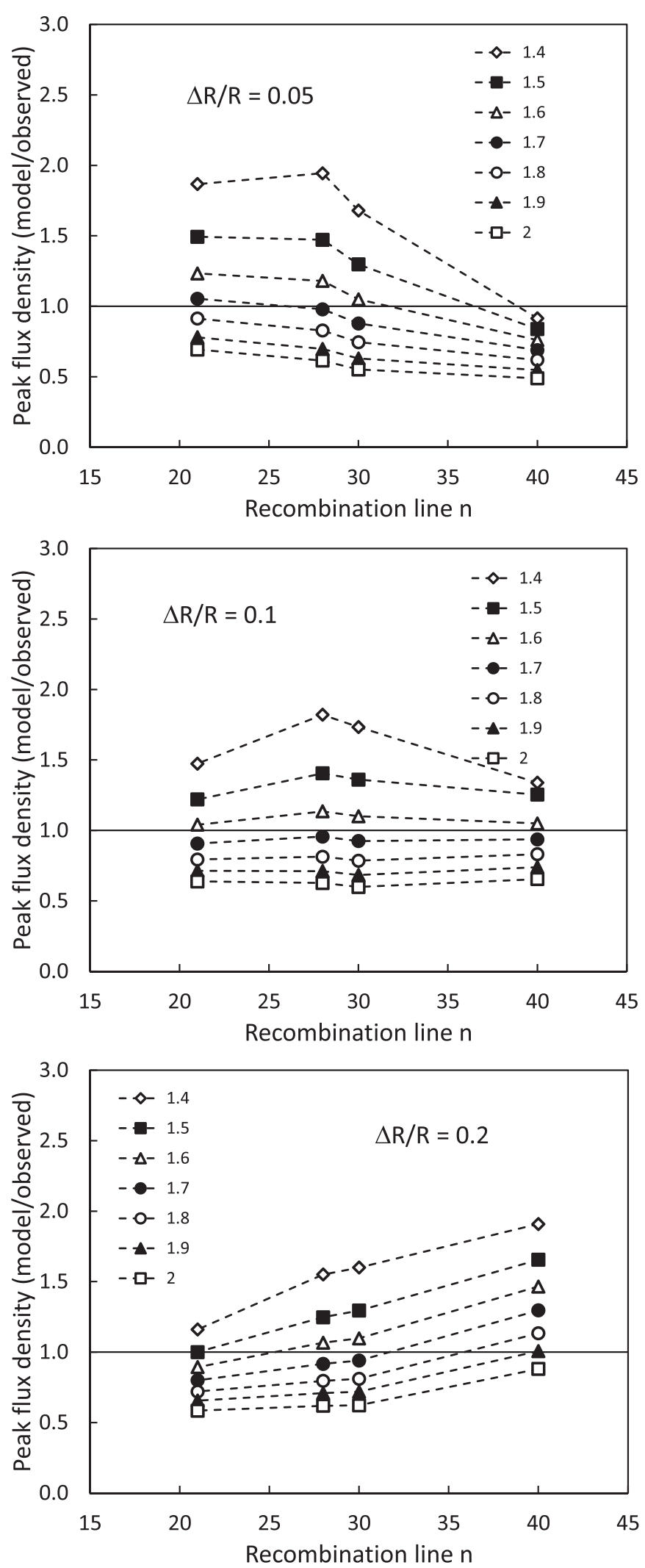

Figure 7. Ratio of model and observed peak flux densities for the recombination lines $\mathrm{H} 40 \alpha, \mathrm{H} 30 \alpha, \mathrm{H} 28 \alpha$, and $\mathrm{H} 21 \alpha$ for electron temperatures ranging from $1.4 \times 10^{4} \mathrm{~K}$ to $2.0 \times 10^{4} \mathrm{~K}$, and three values of the shell width $\Delta R / R: 0.05$, 0.1 , and 0.2 from top to bottom, respectively.

This shell was probably ejected by $\eta$ Carinae in an episode similar to those which formed the Homunculus and the LH, and for that reason, we called it the "Baby Homunculus." 

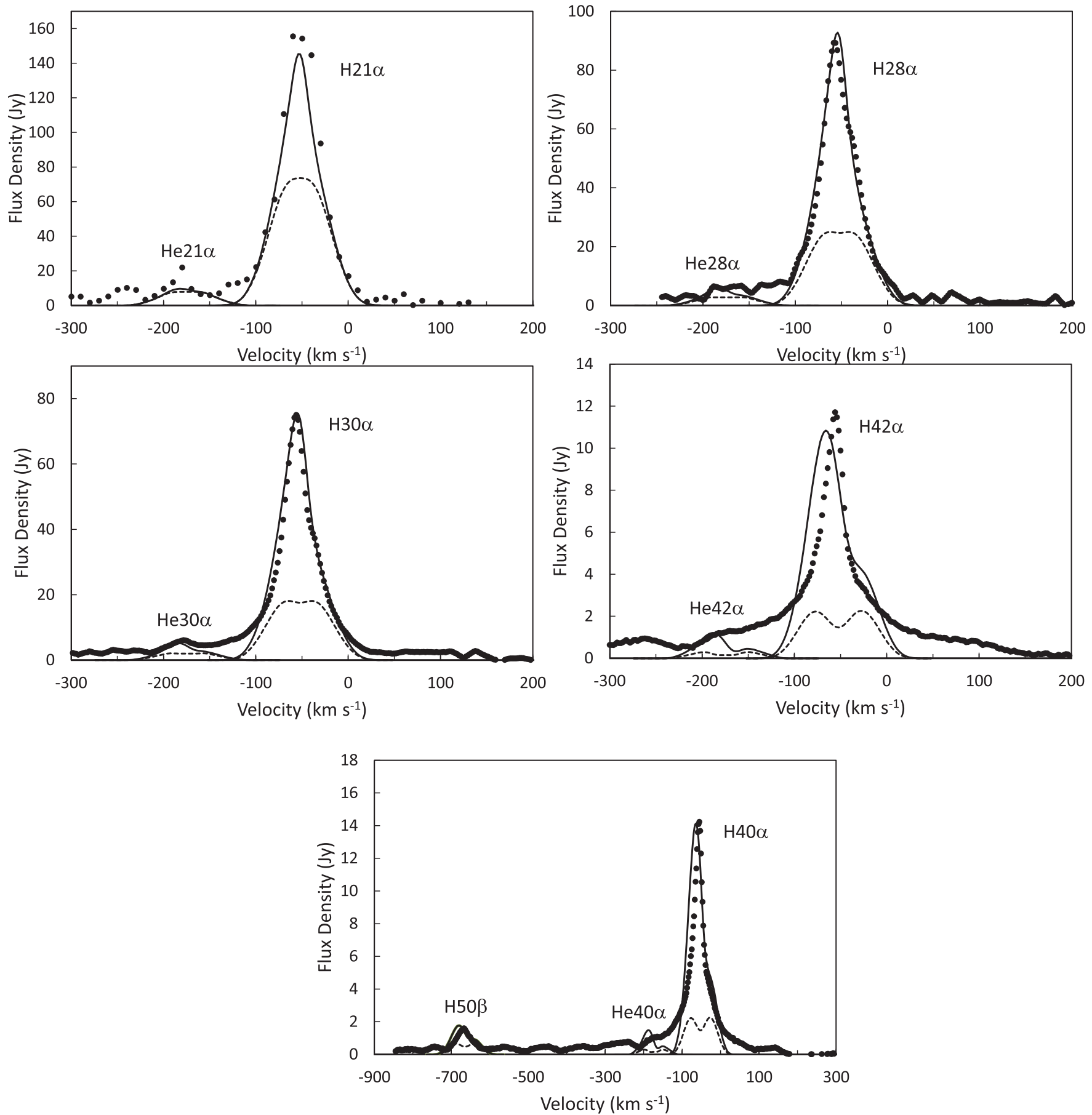

Figure 8. Observed (points) and best-model (solid line) line profiles for the $\mathrm{H} \alpha$ and $\mathrm{He} \alpha$ recombination lines, as well as for the $\mathrm{H} 50 \beta$ line. The dashed line represents the line profiles obtained assuming LTE conditions.

The bulk velocity of the shell may reflect the orbital velocity of $\eta$ Carinae in the direction of the line of sight at the corresponding orbital phase. The ejection epoch is difficult to determine because although we know the size of the shell, our model assumes a gradient in the expansion velocity. However, looking at the historical light curve at optical wavelengths compiled by Fernández-Lajús et al. (2009), shown in Figure 9, we can guess that the ejection occurred around 1941, when there was another sudden increase in stellar luminosity, similar to that which formed the LH. This would require a mean expansion velocity of about $30 \mathrm{~km} \mathrm{~s}^{-1}$ to attain the present size of $0.0022 \mathrm{pc}$.

\subsection{Dynamical Evolution of a Wind-blown Shell}

The origin of $\eta$ Carinae massive bursts is not clear yet, but may be possibly related to the S Dor cycles of luminous hot stars, though much more massive and luminous than those of other LBV stars (Pastorello et al. 2010). LBV variability is categorized by its change in brightness (Humphreys \& Davidson 1994): (1) giant eruptions, with brightness changes of $\Delta m>2$ in timescales of few years; (2) eruptions, with $\Delta m<2$ in timescales of few months up to a couple of years; and (3) small oscillations, with $\Delta m<0.5$, in timescales of a few days to a few weeks. 


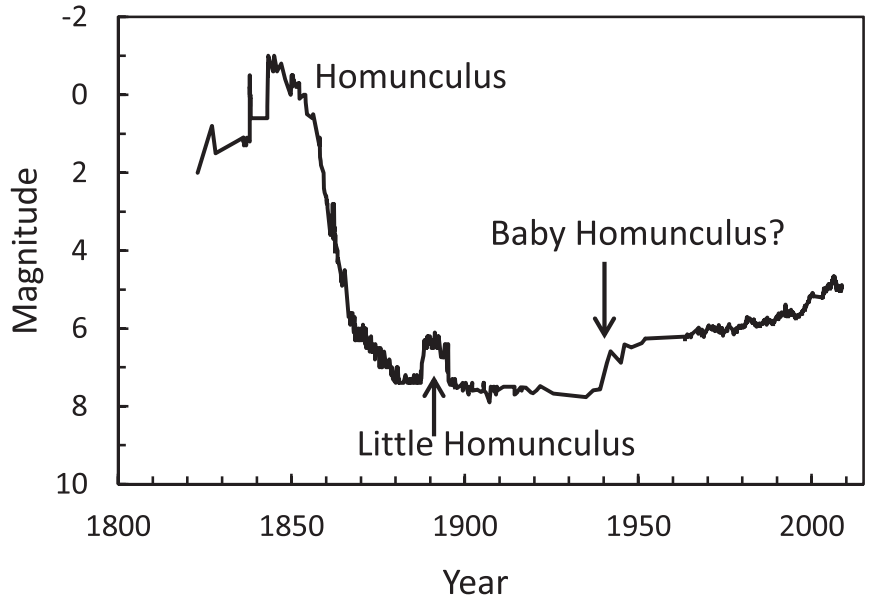

Figure 9. Historical light curve of $\eta$ Carinae, built using data compiled by Fernández-Lajús et al. (2009).

Based on the observed light curve of $\eta$ Carinae shown in Figure 9, the 1940s burst resulted in $\Delta m_{V} \simeq 1$ and may be related to a LBV eruption. A similar case might be that of AG Car during its 1990s eruption, in which its brightness increased by $\Delta m \sim 2$ during a period of almost three years. During the maximum, the star reached a mass-loss rate of $\dot{M} \sim$ $10^{-3.8} M_{\odot} \mathrm{yr}^{-1}$, almost one order of magnitude larger than that at the minimum, though with a reduced wind speed of a few hundred $\mathrm{km} \mathrm{s}^{-1}$ (Stahl et al. 2001).

A similar case might be an increase in the continuous massloss rate and decrease in the wind velocity of $\eta$ Carinae; once the wind mass-loss rate decreased, the wind terminal speed increased again. This two-phased wind evolution could be responsible for the formation of the shell-like structure that expands outward relative to the central object. The internal part would be a low-density expanding cavity created by the fast and less massive post-eruption wind, surrounded by a denser shell expanding at lower speeds, a relic of the massive wind generated during the eruption.

The dynamical evolution of an expanding shell driven by a continuous stellar wind is well described by the classical work of Weaver et al. (1977). According to that work, the radius $R_{1}(t)$ of the expanding shock formed between the wind of velocity $v_{w}$ and mass-loss rate $\dot{M}$ and its surrounding medium of density $\rho_{0}$ is

$$
R_{1}(t) \simeq L_{w}^{1 / 5} \rho_{0}^{-1 / 5} t^{3 / 5},
$$

while the expanding velocity $v_{1}(t)$ would be given by

$$
v_{1}(t) \equiv \dot{R} \simeq \frac{3}{5} L_{w}^{1 / 5} \rho_{0}^{-1 / 5} t^{-2 / 5}
$$

where $L_{w} \simeq \dot{M} v_{w}^{2}$ is the wind luminosity and $t$ is the elapsed time from the start of the eruption. Once the eruption is over, and the star goes back to quiescent state at $t=t_{\text {trans }}$, the ejecta will expand further but without any input of energy at its interior. At this stage, for $t>t_{\text {trans }}$, Equation (1) above is no longer valid. The dynamics of the expanding shell will then be better described as a blast wave. Here, two different phases are possible depending on the cooling efficiency of the shocked gas. The first is the Sedov solution for an adiabatically expanding shell, while the second is a momentum conserved snow-plow shell. The typical cooling timescale is given as $\tau_{\text {cool }} \simeq k T / n \Lambda \sim 5 \times 10^{-3} n^{-1} \mathrm{yr}$, where $\Lambda$ is the energy loss rate, $T \sim 10^{5} \mathrm{~K}$, and the number density $n$ is given in $\mathrm{cm}^{-3}$.

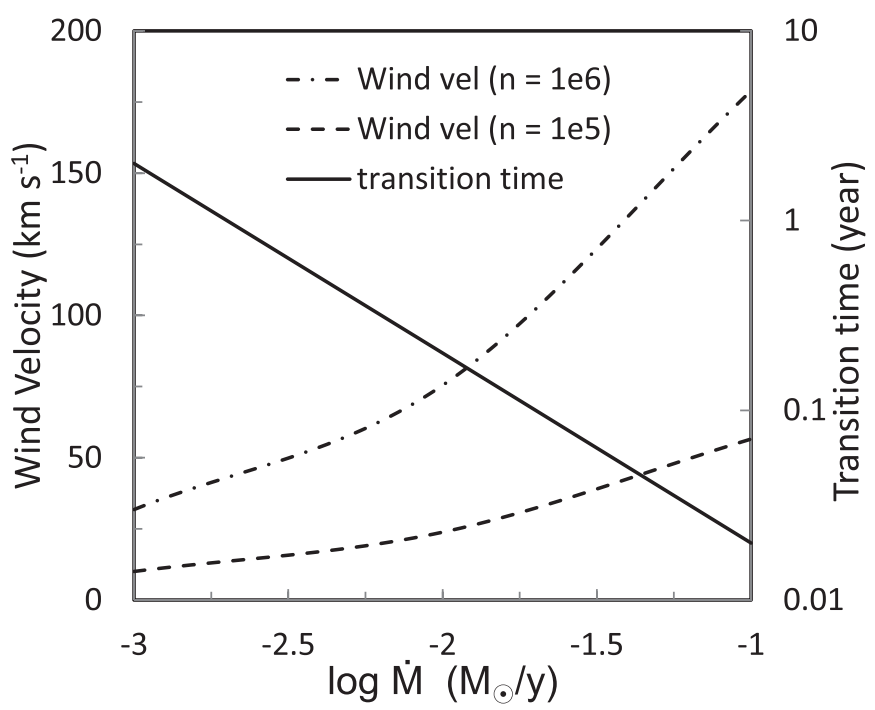

Figure 10. Wind velocity (left axis) for two values of the ambient density: $10^{5} \mathrm{~cm}^{-3}$ (dash line) and $10^{6} \mathrm{~cm}^{-3}$ (dot-dash line), and duration of the LBV outburst (right axis, solid line) as a function of the mass-loss rate during the outburst.

In the case of $\eta$ Carinae, the whole process occurs in highdensity regimes (the stellar wind and surrounding medium), i.e., $n>10^{3} \mathrm{~cm}^{-3}$. Therefore, we may consider the expansion of the post-eruption shell in the radiative (snow-plow) regime as

$$
R_{2}(t)=R_{\text {trans }}\left(\frac{t}{t_{\text {trans }}}\right)^{1 / 4}
$$

and

$$
v_{2}(t)=v_{\text {trans }}\left(\frac{t}{t_{\text {trans }}}\right)^{-3 / 4},
$$

where $v_{\text {trans }}$ stands for the velocity at the transition time which can be obtained from Equations (1) and (2).

The mass of the shell $M_{\text {shell }}$ gives an upper limit to the mass ejected by the star:

$$
M_{\text {shell }}=\mu m_{H} N_{e} \pi R_{2}^{2}\left(t_{2}\right) \Delta R_{2}=\dot{M} t_{\text {trans }},
$$

where $t_{2}$ is the time elapsed since the beginning of the ejection until the observation; it was taken at $71 \mathrm{yr}$, linking the beginning of the ejection to the luminosity increase observed in 1941.

Using Equations (1)-(5) and our shell model parameters, we obtain

$$
\begin{gathered}
t_{\text {trans }}=\frac{0.002}{\dot{M}}, \\
v_{w}^{2}=0.18 \dot{M}^{3 / 4} N_{0},
\end{gathered}
$$

and

$$
v_{2}\left(t_{2}\right)=\frac{3}{5} \frac{R_{2}\left(t_{2}\right)}{t_{2}},
$$

where $\dot{M}$ is given in $M_{\odot} \mathrm{yr}^{-1}, t_{\text {trans }}$ in years, $N_{0}$ in $\mathrm{cm}^{-3}$, and $v_{w}$ in $\mathrm{km} \mathrm{s}^{-1}$.

From Equation (8), we immediately obtain $v_{2}\left(t_{2}\right)=$ $19 \mathrm{~km} \mathrm{~s}^{-1}$, which is in excellent agreement with the expansion velocity derived for the shell. The wind velocity during the eruption and its duration versus the mass-loss rate is presented in Figure 10 for two values of the ambient density: $10^{5}$ and $10^{6} \mathrm{~cm}^{-3}$. It is clear that the wind velocities and mass-loss rates are compatible with what is expected in an LBV eruption. 
In spite of the fact that we cannot strictly constrain the wind parameters during the eruption, the model used to fit the observed data gives reasonable values, compared to those expected for LBV eruptions, i.e., wind velocities of $v_{w}=$ $50-200 \mathrm{~km} \mathrm{~s}^{-1}$ and mass-loss rates of $\dot{M}=10^{-2.5}-10^{-1.5} M_{\odot}$ $\mathrm{yr}^{-1}, \sim 10-100$ times larger than the current value estimated for $\eta$ Carinae, lasting between one year and one month, respectively.

\subsection{The High-excitation Optical Lines and the Weigelt Blobs}

The densities and temperatures of the shell model also predict the existence of high-excitation optical emission lines, which were in fact observed in the $0^{\prime} .1-0^{\prime \prime} 3$ surroundings of $\eta$ Carinae (Mehner et al. 2010). Spatial maps of these lines show the presence of compact condensations, known as the Weigelt blobs, which present lines of highly ionized ions like [Ne III] and [Fe III]; their presence requires densities of $N_{e} \geqslant 10^{7} \mathrm{~cm}^{-3}$ and temperatures of $T_{e} \sim 2 \times 10^{4} \mathrm{~K}$. Their line-of-sight velocities are small relative to the wind velocity $\left(-47 \mathrm{~km} \mathrm{~s}^{-1}\right.$ in the geocentric reference frame or $-58 \mathrm{~km} \mathrm{~s}^{-1}$ in the LSR), but they were never related to the source of the radio recombination lines observed by Cox et al. (1995a).

Although these condensations are known since 1986 (Weigelt \& Ebersberger 1986), their nature remained a puzzle. As a consequence of our study, it is possible that the Weigelt blobs are part of the Baby Homunculus; not only their velocities coincide, but also their densities and temperatures.

The behavior of the radio emission and the optical light curve of the Weigelt blobs along the different phases of the binary cycle are also similar. In fact, Mehner et al. (2010) reported that the optical light curve of the high-excitation lines increased up to the middle of the binary phase between 1988 and 2003.5, presenting another maximum before the sharp minimum, which was also observed in the radio light curve (Abraham et al. 2005). This behavior can be understood if the shell is ionized by the secondary star. During the minimum, the ionizing photons do not reach the high density shell and the plasma recombines with time scales of days. When the ionizing photons reach the Baby Homunculus again, they ionize the gas, but the ionizing front propagates slower than the recombination timescale, explaining the gradual increase in the observed flux density.

Other characteristics of the Weigelt blobs can be explained if they are part of the expanding shell, such as the $10 \mathrm{~km} \mathrm{~s}^{-1}$ difference in velocity between the high-ionization lines and those of the low-excitation and low-ionization levels found by Smith (2004), which can be a consequence of the velocity gradient across the shell.

The ejection time of the Weigelt blobs is also controversial, ranging from 1890 (Smith 2004) to 1941 (Dorland et al. 2004); the last one compatible with our interpretation that the shell was ejected in 1941.

\section{CONCLUSIONS}

In this paper, we presented millimeter and submillimeter observations of the continuum emission and $\mathrm{H}$ and $\mathrm{He}$ recombination lines of $\eta$ Carinae obtained with ALMA in 2012 November.

The continuum spectrum is characteristic of thermal bremsstrahlung, increasing to a frequency up to $230-280 \mathrm{GHz}$, a frequency at which the source became optically thin, and decreasing slowly at higher frequencies. The high flux density observed in the recombination lines requires NLTE conditions, and the presence of the He lines indicate that the ionizing star should have a surface temperature higher than that attributed to $\eta$ Carinae.

We modeled the emitting region as a spherical shell, moving in the direction of the observer with a bulk velocity of $-52 \mathrm{~km} \mathrm{~s}^{-1}$ and expanding with velocities ranging between 20 and $60 \mathrm{~km} \mathrm{~s}^{-1}$. These velocities were needed to reproduce the observed line profiles. The continuum spectrum and the intensity of the recombination lines could be better reproduced by a shell of radius $R=0.0022 \mathrm{pc}$, width $\Delta R=0.1 R$, electron temperature $T=1.7 \times 10^{4} \mathrm{~K}$, and electron density $N_{e}=1.25 \times$ $10^{7} \mathrm{~cm}^{-3}$. The results of this model could be checked with future observations, when longer baselines and a larger number of ALMA antennae become available.

The physical parameters of the shell and the behavior of the radio light curve along the orbital phase are similar to those of the Weigelt blobs, and we suggest that these blobs are part of the shell. We also suggest that the shell was ejected around 1941, when there was a sudden increase in the optical luminosity of $\eta$ Carinae. As this increase is similar to that observed when the LH was formed, we proposed that the newly discovered one shall be known as the "Baby Homunculus."

This work was supported by the Brazilian agencies CAPES, CNPq, and FAPESP. D.F.G. thanks the European Research Council (ADG-2011 ECOGAL) and Brazilian agencies CNPq (No. 300382/2008-1), CAPES (No. 3400-13-1), and FAPESP (No. 2011/12909-8) for financial support.

\section{APPENDIX A BREMSSTRAHLUNG}

Let us consider an ionized source with spherical symmetry, formed by a shell of radius $R$, width $\Delta R$, and constant electron density and temperature. The source is divided in rectangular cells of size $(\Delta x, \Delta y)$ in the plane of the sky, and the radiation transfer equation is solved along the line of sight ( $z$ coordinate) in each cell.

The resulting continuum flux density $S_{v}^{\mathrm{c}}$ is given by

$$
S_{v}^{c}(x, y)=\frac{2 \pi v^{2}}{c^{2}} k T_{e}\left[1-\exp \left(-\tau_{v}^{c}(x, y)\right)\right] \frac{\Delta x \Delta y}{D^{2}}
$$

where $T_{e}$ is the electron temperature, $v$ the frequency, $k$ the Boltzmann constant, $\tau_{v}^{c}(x, y)$ the optical depth for free-free absorption integrated along the line of sight, and $D$ the distance from the source to the observer.

Since we assumed constant electron temperature and density in the emitting region, the optical depth can be written as $\tau_{v}^{c}(x, y)=\kappa_{v}^{c} \ell(x, y)$, where $\ell(x, y)$ is the depth of the absorbing region along the line of sight, and the absorption coefficient for free-free radiation $\kappa_{v}^{c}$ is given by (Brocklehurst \& Seaton 1972)

$$
\kappa_{v}^{c}=6.94 \times 10^{-8} \frac{N_{e} N_{i}}{v^{2}}\left(\frac{10^{4}}{T_{e}}\right)^{3 / 2} g_{\mathrm{ff}} \mathrm{pc}^{-1}
$$

where $N_{e}$ and $N_{i}$ are the electron and ion number densities and $g_{\text {ff }}$ is the Gaunt factor, given by

$$
g_{\mathrm{ff}}=\left(4.69+1.5 \ln T_{e}-\ln v\right) .
$$




\section{APPENDIX B}

\section{RECOMBINATION LINES}

The total (continuum plus line) flux density $S_{v}^{1+c}(x, y)$ of each cell can be obtained from

$$
\begin{aligned}
S_{v}^{l+c}(x, y)= & \frac{2 \pi v^{2}}{c^{2}} k T_{e} \frac{\Delta x \Delta y}{D^{2}} \\
& \times \int_{0}^{\ell(x, y)}\left(\kappa_{v}^{c}+b_{n+m} \kappa_{v, \text { LTE }}^{l}\right) \exp \left(-\tau_{v}^{l+c}\right) d z,
\end{aligned}
$$

where

$$
\tau_{v}^{l+c}(x, y, z)=\int_{0}^{z}\left(b_{n} \beta_{n, n+m} \kappa_{v, \mathrm{LTE}}^{l}+\kappa_{v}^{c}\right) d z^{\prime} .
$$

$\kappa_{v}^{c}$ is given by Equation (A2), $\kappa_{v, \text { LTE }}^{l}$ is the LTE line absorption coefficient for the transition between levels with quantum numbers $n$, and $n+m$ is given by (Brocklehurst $\&$ Seaton 1972)

$$
\begin{aligned}
\kappa_{\nu, \mathrm{LTE}}^{l}= & 1.064 \times 10^{-12} \frac{N_{e} N_{+}}{v}\left(\frac{10^{4}}{T}\right)^{2.5} m K(m) \\
& \times \exp \left(\chi_{n} / k T\right)\left(\nu \phi_{\nu}\right)
\end{aligned}
$$

where $N_{+}$is the number of ions $\left(\mathrm{H}^{+}\right.$or $\left.\mathrm{He}^{+}\right), m K(m)$ is related to the oscillator strength (Menzel 1968), with values of 0.1908 for $m=1$ and 0.05266 for $m=2, \chi_{n}$ is the energy of level $n$, $b_{n}$ is the population of level $n$ relative to its population in LTE, calculated by Storey \& Hummer (1995) for several values of $n$, $N_{e}$, and $T_{e}$,

$$
\beta_{n, n+m}=\frac{1-\left(b_{n+m} / b_{n}\right) \exp (-h v / k T)}{1-\exp (-h v / k T)},
$$

$\phi_{\nu}$ is the normalized line profile, which can be calculated from

$$
\phi_{v}=\int_{-\infty}^{\infty} \phi_{\nu-v^{\prime}+v_{0}}^{P} \phi_{v^{\prime}}^{D} d v^{\prime}
$$

where $\phi_{v}^{D}$ represents the Doppler-broadening profile, given by (Brocklehurst \& Leeman 1971)

$$
\phi_{v}^{D}=\frac{\alpha}{\pi^{1 / 2} v_{0}} \exp \left\{-\left[\alpha\left(v-v_{0}\right) / v_{0}\right]^{2}\right\}
$$

with $\alpha=\left[m_{i} c^{2} /\left(2 k T_{D}\right]^{1 / 2}\right.$, where $m_{i}$ is the mass of the ion and $T_{D}$ is the Doppler temperature, which includes the thermal and turbulent velocities; $v_{0}$ is the rest frequency of the transition.

$\phi_{v}^{P}$ represents the pressure-broadening profile, which can be written as

$$
\phi_{\nu}^{P}=\frac{\delta}{\pi} \frac{1}{\left(v-v_{0}\right)^{2}+\delta^{2}},
$$

with $\delta=1 / 2 \pi\langle v Q\rangle N_{e}$, where $\langle v Q\rangle$ is the total inelastic crosssection averaged over the Maxwellian distribution (Brocklehurst \& Leeman 1971).
The width of the thermal line can be calculated from

$$
(\Delta v)^{D}=7.13 \times 10^{-5} c\left[\frac{m_{H}}{m_{i}}\left(\frac{T_{D}}{10^{4}}\right)\right]^{1 / 2},
$$

which corresponds to $21 \mathrm{~km} \mathrm{~s}^{-1}$ for a $10^{4} \mathrm{~K}$ ionized $\mathrm{H}$ plasma.

The width of the pressure-broadened line can be calculated from (Báez-Rubio et al. 2013)

$$
(\Delta v)^{P}=8 \times 10^{-10} \frac{n^{5} N_{e} c}{v Z^{2} T_{e}^{0.1}} n \leqslant 30 .
$$

$$
(\Delta v)^{P}=6.7 \times 10^{-9} \frac{n^{4.6} N_{e} c}{v Z^{2} T_{e}^{0.1}} 30<n<100
$$

which corresponds to $2.5 \mathrm{~km} \mathrm{~s}^{-1}$ for $n=42, T_{e}=10^{4}$ $\mathrm{K}$, and $N_{e}=10^{7} \mathrm{~cm}^{-3}$ and only $5 \times 10^{-3} \mathrm{~km} \mathrm{~s}^{-1}$ for $n=21$, in both cases, much smaller than the thermal width, and for that reason, pressure broadening was not included in the calculations.

\section{REFERENCES}

Abraham, Z., Falceta-Gonçalves, D., Dominici, T. P., et al. 2005, A\&A, 437, 997

Báez-Rubio, A., Martin-Pintado, J., Thum, C., \& Planesas, P. 2013, A\&A, 553, A45

Brocklehurst, M., \& Leeman, S. 1971, ApL, 9, 35

Brocklehurst, M., \& Seaton, M. F. 1972, MNRAS, 157, 179

Corcoran, M. F., Ishibashi, K., Swank, J. H., \& Petre, R. 2001, ApJ, 547, 1034

Cox, P., Martin-Pintado, J., Bachiller, R., et al. 1995a, A\&A, 295, L39

Cox, P., Mezger, P. G., Sievers, A., et al. 1995b, A\&A, 297, 168

Damineli, A. 1996, ApJL, 460, L49

Dorland, B. N., Currie, D. G., \& Hahan, A. R. 2004, AJ, 127, 1058

Duncan, R. A., White, S. M., \& Lim, J. 1997, MNRAS, 290, 680

Fenández-Lajús, E., Fariña, C., Torres, A. F., et al. 2009, A\&A, 493, 1093

Gaviola, E. 1950, ApJ, 111, 408

Humphreys, R. M., \& Davidson, K. 1979, ApJ, 232, 409

Humphreys, R. M., \& Davidson, K. 1994, PASP, 106, 1025

Humphreys, R. M., Davidson, K., \& Smith, N. 1999, PASP, 111, 1124

Ishibashi, K., Gull, T. R., Davidson, K., et al. 2003, AJ, 125, 3222

Mehner, A., Davidson, K., Ferland, G. J., \& Humphreys, R. M. 2010, ApJ, 710,729

Menzel, D. H. 1968, Natur, 218, 756

Morris, P. W., Waters, L. B. F. M., Barlow, M. J., et al. 1999, Natur, 402, 502

Pastorello, A., Botticella, M. T., Trundle, C., et al. 2010, MNRAS, 408, 181

Smith, N. 2004, MNRAS, 351, L15

Smith, N 2005, MNRAS, 357, 1330

Smith, N. 2011, MNRAS, 415, 2020

Smith, N., \& Gehrz, R. D. 1998, AJ, 116, 823

Smith, N., Gehrz, R. D., Hinz, P. M., et al. 2003, AJ, 125, 1458

Smith, N., Li, W., Silverman, J. M., Ganeshalingam, M., \& Filippenko, A. V. 2011, MNRAS, 415, 773

Stahl, O., Jankovics, I., Kovács, J., et al. 2001, A\&A, 375, 54

Storey, P. J., \& Hummer, D. G. 1995, MNRAS, 272, 41

Teodoro, M., Damineli, A., Sharp, R. G., Groh, J. H., \& Barbosa, C. L. 2008, MNRAS, 387, 564

Weaver, R., McCray, R., Castor, J., Shapiro, P., \& Moore, R. 1977, ApJ, 218, 377

Weigelt, G., \& Ebersberger, J. 1986, A\&A, 163, L5 